\title{
Réponse de la culture de soja (Glycine max L. (Merril) à l'apport des biomasses vertes de Tithonia diversifolia (Hemsley) A. Gray comme fumure organique sur un Ferralsol à Lubumbashi, R.D. Congo
}

\begin{abstract}
Kasongo Lenge Mukonzo Emery(a), Mwamba Mulembo Théodore(a), Tshipoya Masumbuko Patient(b), Mukalay Muamba Joseph(a), Useni Sikuzani Yannick(c)*, Mazinga Kwey Michel (d), Nyembo Kimuni Luciens (c)

(a) Laboratoire de pédologie, Faculté des sciences agronomiques, Université de Lubumbashi BP 1825, Lubumbashi, RD Congo; (b) Chercheur au laboratoire de pédologie, Faculté des sciences agronomiques, Université de Lubumbashi BP 1825, Lubumbashi, RD Congo; (c) Département de phytotechnie, Faculté des sciences agronomiques, Université de Lubumbashi BP 1825, Lubumbashi, RD Congo ; (d) Laboratoire des cultures in vitro, Département de phytotechnie, Faculté des sciences agronomiques, Université de Lubumbashi BP 1825, Lubumbashi, RD Congo

* auteur pour correspondance : yannickusen@gmail.com ; Tél : +243813666582

Original submitted in on $7^{\text {th }}$ January 2013. Published online at www.m.elewa.org on 30th March 2013.
\end{abstract}

\section{RÉSUMÉ}

Objectifs : cette étude a été initiée en vue d'évaluer les effets des apports des biomasses vertes de Tithonia diversifolia sur le comportement du soja installé sur un ferralsol à Lubumbashi.

Méthodologie et résultats : l'essai a été conduit à la ferme Kasapa de l'université de Lubumbashi $\left(11^{\circ} 36^{\prime} \mathrm{S}\right.$ et $27^{\circ} 18 \mathrm{E}$, sur une altitude moyenne de $1243 \mathrm{~m}$, dans la zone périurbaine de Lubumbashi) et installé suivant un dispositif en blocs complets randomisés. Les traitements, en quatre répétitions, ont été constitués d'un témoin (TO), de trois niveaux d'apport de biomasses fraiches de Tithonia diversifolia à savoir T1, T2 et T3 comprenant respectivement $3381 \mathrm{~kg}, 6762 \mathrm{~kg}$ et $10142 \mathrm{~kg}$ de biomasse de Tithonia diversifolia par hectare ; ainsi que d'un apport d'engrais minéral NPK 10-20-10 (T4). Les résultats obtenus ont montré que le rendement le plus élevé a été observé sur les parcelles ayant reçu $10143 \mathrm{~kg}$ de biomasse de Tithonia diversifolia par hectare comme mulch, avec une moyenne de $3101 \mathrm{~kg}$ à l'hectare. La plus grande quantité de biomasse de Tithonia diversifolia (T3) a induit un rendement supérieur à la dose d'engrais minéral appliquée.

Conclusion et principales applications de la recherche : dans la zone périurbaine de Lubumbashi, l'apport des biomasses de Tithonia diversifolia (10143 kg /hectare) comme fertilisant constituerait un atout majeur pour l'accroissement des rendements du soja dans un contexte de dégradation des sols et de prix élevé des engrais minéraux.

Mots clés : biomasse de Tithonia diversifolia, fumure organique, soja, Ferralsol, Lubumbashi 
Response of soybean (Glycine max L. (Merril) to the green biomass of Tithonia diversifolia (Hemsley) A. Gray used as organic fertilizer on a Ferralsol in Lubumbashi, DR Congo

\section{Abstract}

Objectives: This study was initiated to evaluate the effects of the green biomass of Tithonia diversifolia used as mulch on the behavior of soybean on a Ferralsol in Lubumbashi.

Methodology and Results: The trial was conducted at the farm Kasapa University of Lubumbashi $\left(11^{\circ} 36^{\prime}\right.$ $S$ and $27^{\circ} 18^{\prime} \mathrm{E}$, on an average altitude of $1243 \mathrm{~m}$, in the suburbs of Lubumbashi) using a randomized complete block method. Treatments in four replications were made by a control (T0), three levels of intake of fresh biomass of Tithonia diversifolia namely T1, T2 and T3 respectively including $3381 \mathrm{~kg}, 6762 \mathrm{~kg}$ and $10142 \mathrm{~kg}$ of biomass Tithonia diversifolia per hectare and a mineral fertilizer NPK 10-20-10 (T4). The results showed that the highest yield was observed on plots T3 that had received $10143 \mathrm{~kg}$ of biomass per hectare as Tithonia diversifolia mulch, with an average yield of $3101 \mathrm{~kg}$ hectare. Biomass of Tithonia diversifolia induced a higher yield than the dose of chemical fertilizer applied.

Conclusion and main applications of the research: the contribution of Tithonia diversifolia as fertilizer is a major asset for increasing yields of food legumes in the suburbs of Lubumbashi, in the context of land degradation and high prices of mineral fertilizers.

Keywords: biomass of Tithonia diversifolia, organic manure, soybean, Ferralsol, Lubumbashi

\section{INTRODUCTION}

La fertilité ou richesse d'un agro-écosystème se mesure par sa capacité à produire de manière performante différents produits utiles à l'homme. Le maintien de cette performance est vital tandis que sa baisse se traduit par une perte progressive des rendements. Généralement, par le travail du sol et l'ajout des substances nutritives, l'agriculteur compense la perte d'énergie causée par les récoltes ou par d'autres facteurs dans le but d'améliorer le rendement de son agro-écosystème et la productivité de son travail (Mbonigaba, 2007). $\mathrm{Si}$ cela est savamment réalisé en agriculture moderne à travers l'emploi des fumures et amendements adéquats et souvent couteux, l'agriculture traditionnelle pratiquée par la plupart des ménages ruraux d'Afrique subsaharienne s'appuie sur la résilience naturelle des terres à travers leur mise en jachère (Kaho et al., 2011). Pourtant, la pression démographique sur les terres réduit sensiblement la durée de ces jachères et partant, la capacité de régénération naturelle de la fertilité des sols. Cela entretient un processus de dégradation des sols dont les conséquences sur la sécurité alimentaire et les revenus des ménages sont graves (Chukwuka et Omotayo, 2009). La recherche des alternatives porteuses de soulagement est très importante. Dans un contexte d'insécurité alimentaire, de réduction de la fertilité des sols et de la hausse des prix des engrais sur les marchés, il apparaît nécessaire d'utiliser pour l'agriculture les nutriments disponibles et à faible coût. De plus, l'application exclusive des engrais minéraux n'est généralement efficace que pendant les premières années d'apports continus ; on constate en effet une baisse de rendement après quelques années à cause de la dégradation des propriétés des sols (Useni et al., 2012). Pourtant, les biomasses de certaines espèces végétales invasives et de recolonisation, comme Tithonia diversifolia, sont reconnues comme étant pourvoyeuses des éléments minéraux aux sols pauvres, augmentant ainsi les rendements (Jamaa et al., 2000). Des récentes recherches ont montré que les biomasses de Tithonia diversifolia ont augmenté les rendements du maïs au Kenya (Niang et al., 1996; Jamaa et al., 2000), au Zimbabwe (Jirri et Waddington, 1998), au Malawi (Ganunga et al., 1998) et au Cameroun (Kaho et al., 2011). Des quantités croissantes de biomasses de Tithonia diversifolia ont été appliquées comme mulch sur un sol acide. La réponse de la culture du soja a été évaluée par rapport à cette fumure en comparaison avec une fumure minérale à base de l'azote, le phosphore et le potassium d'une part, et 
d'autre part avec un témoin non fertilisé. Le choix de Tithonia diversifolia a été motivé par sa dominance comme plante de recolonisation dans la zone d'étude tandis que la culture de soja a été choisie pour son importance comme source alternative des protéines dans la diète des

\section{MATERIEL ET METHODES}

Description du site d'étude : Cette étude a été conduite à la ferme Kasapa, à $11^{\circ} 36^{\prime} \mathrm{S}$ et $27^{\circ} 18$ $\mathrm{E}$, sur une altitude moyenne de $1243 \mathrm{~m}$, dans la banlieue de Lubumbashi, au Sud-est de la République Démocratique du Congo (Figure 1). L'essai a été installé sur un bloc ayant porté une longue monoculture du maïs au cours des années antérieures. Sa flore de recolonisation est dominée par des espèces telles que Imperata cylindrica, Cynodon dactylon et Tithonia diversifolia qui occupent le terrain à des degrés de recouvrement variés. La couverture pédologique est du type ménages pauvres. Le but était d'apprécier la réponse de la culture de soja à l'application de ces matériels dans la perspective de leur utilisation comme produits de substitution aux engrais chimiques généralement trop coûteux pour les pauvres.

ferralitique avec un $\mathrm{pH}$ à l'eau oscillant autour de 5,2 . La région de Lubumbashi est caractérisée par un climat du type Cw6 de la classification de Koppën (FAO, 2005). Elle est caractérisée par une période de croissance normale (Figure 2) d'une durée moyenne de 182 jours en moyenne constituée une période humide d'environ 150 jours (FAO, 2005). Cette période de croissance commence à la seconde moitié d'octobre pour s'arrêter vers la mi-avril tandis que la période humide va de la première moitié de novembre jusqu'à la première décade d'avril (Figure 2).

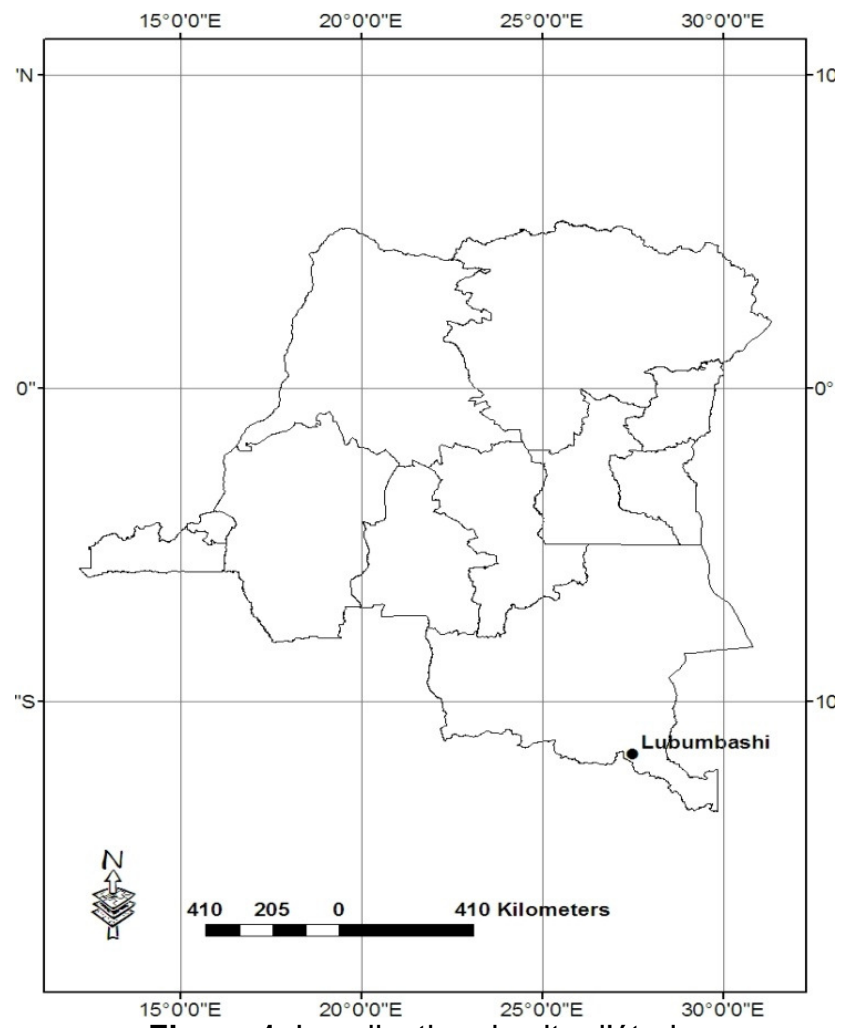

Figure 1. Localisation du site d'étude 


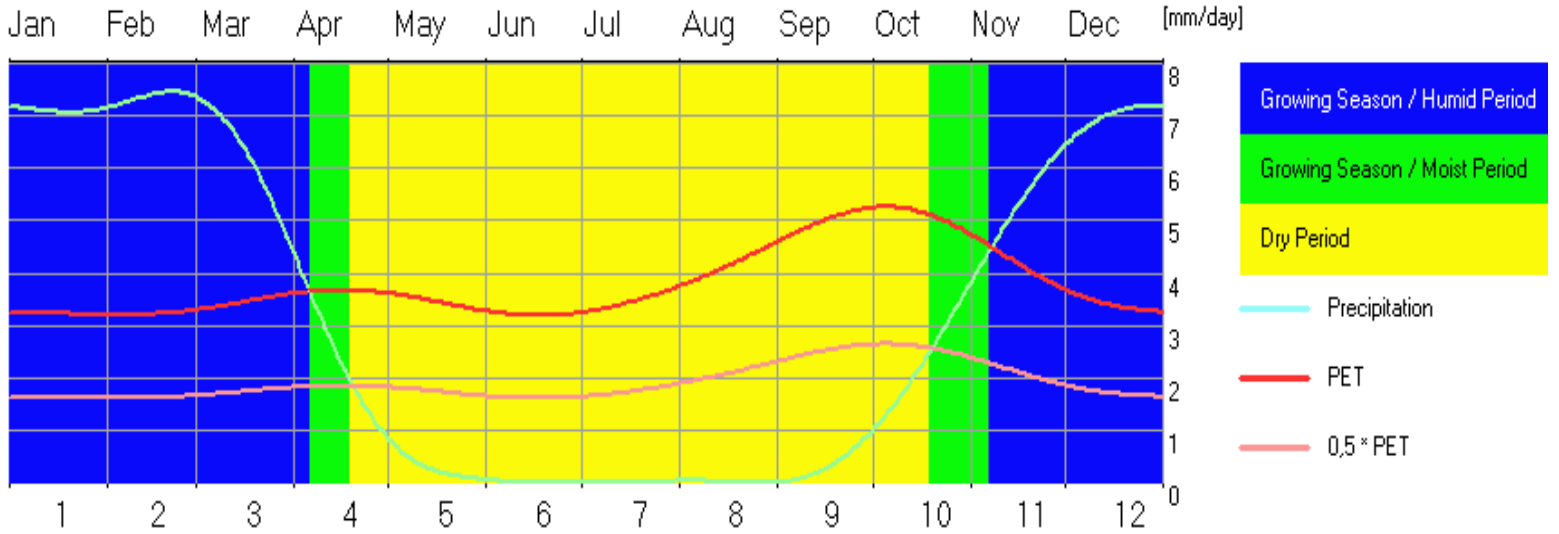

Figure 2 : Période de croissance des cultures à Lubumbashi (FAO, 2005)

Description de l'essai : L'essai a été installé selon un dispositif en blocs complets randomisés portant cinq traitements et quatre répétitions. Les traitements ont été constitués d'un témoin (T0), de trois niveaux d'apport de biomasses fraiches de Tithonia diversifolia $(3,48 \% \mathrm{~N}, 0,8 \% \mathrm{P}$ et $6,57 \%)$ par parcelle, à savoir : T1, T2 et T3 comprenant respectivement $3381 \mathrm{~kg}, 6762 \mathrm{~kg}$ et $10142 \mathrm{~kg}$ de biomasse de Tithonia diversifolia par hectare; ainsi que d'un apport de $100 \mathrm{~kg}$ d'engrais minéral NPK 10-20-10 (T4) constitué des trois éléments majeurs, tels que recommandé en culture de soja (Javaheri et Baudoin, 2001). Les parties tendres (feuilles et tiges vertes) de la biomasse fraiche de Tithonia diversifolia, recueillies des touffes colonisant le site d'expérimentation ont été utilisées comme mulch. Les parcelles élémentaires de l'essai ont été dimensionnées à la superficie de $2 \mathrm{~m}^{2}$ chacune. Le semis du soja a été réalisé manuellement aux écartements de $40 \mathrm{~cm} \times 20 \mathrm{~cm}$ à raison deux graines par poquet à la date du 24

\section{RESULTATS}

Influence des traitements sur le comportement de la culture: Les valeurs moyennes de ces différentes variables sont consignées dans le tableau 1. Les apports au sol des biomasses de Tithonia diversifolia et de la fumure minérale ont significativement influencé le niveau de rendement de la culture du soja $(p<0,05)$, la hauteur de ses décembre 2011 pour faire coïncider la récolte avec le début de la période sèche. Le traitement statistique des données a consisté en une analyse de la variance couplée au test de Tukey en utilisant le logiciel Minitab 16. Ainsi, la réponse de la culture du soja a été appréciée à travers la comparaison des moyennes des traitements par rapport aux variables rendement de la culture (kg/ha), nombre de gousses par plant, hauteur moyenne des plants et poids de 1000 graines à la maturité de la culture. En outre, la tendance du rendement et du nombre de gousses par plant en fonction des quantités de biomasses de Tithonia diversifolia appliquées a été analysée dans la perspective de l'estimation future des quantités adéquates à appliquer pour ce type d'apport. La variété TGX1880-3E du germoplasme de l'INERA- Kipopo a été utilisée comme matériel végétal. C'est une variété dont le cycle végétatif varie entre 100 et 110 jours.

plants $(p<0,05)$ et le nombre de gousses produits par plant $(p<0,05)$. Néanmoins, la qualité des graines telle que reflétée par le calibre de leurs graines à travers la variable poids de 1000 graines n'a pas été significativement affectée $(p=0,507)$. 
Kasongo et al.... J. Appl. Biosci. 2013.

Tableau 1. Synthèse de la comparaison des moyennes des variables observées par l'ANOVA à un seul critère

\begin{tabular}{|c|c|c|c|c|}
\hline \multirow[b]{2}{*}{ Traitements } & \multicolumn{4}{|c|}{ Variables observées } \\
\hline & $\begin{array}{c}\text { Rendement } \\
(\mathrm{kg} / \mathrm{ha})\end{array}$ & $\begin{array}{c}\text { Nombre de gousses } \\
\text { par plant }\end{array}$ & $\begin{array}{c}\text { Hauteur des } \\
\text { plants }(\mathrm{cm})\end{array}$ & $\begin{array}{c}\text { Poids de } 1000 \\
\text { graines }(\mathrm{g})\end{array}$ \\
\hline TO & $1952,1 \pm 112,0 \mathrm{c}$ & $59,9 \pm 1,88 c$ & $37,88 \pm 0,72 b$ & $88,50 \pm 5,74 a$ \\
\hline T1 & $2619,0 \pm 68,3 b$ & $73,08 \pm 2,08 b$ & $41,80 \pm 2,59 a b$ & $94,00 \pm 9,13 a$ \\
\hline T2 & $2684,3 \pm 111,3 b$ & $79,16 \pm 2,42 a b$ & $43,80 \pm 2,32 a$ & $96,25 \pm 13,82 a$ \\
\hline T3 & $3101,8 \pm 194,8 a$ & $81,4 \pm 3,38 a$ & $44,67 \pm 1,68 \mathrm{a}$ & $102,00 \pm 11,34 a$ \\
\hline $\mathrm{T} 4$ & $2595,9 \pm 78,2 b$ & $78,28 \pm 5,85 a b$ & $45,58 \pm 1,65 a$ & $94,50 \pm 10,34 a$ \\
\hline Niveau de $p$ & $p=0,000$ & $p=0,000$ & $p=0,000$ & $p=0,507$ \\
\hline $\begin{array}{l}\text { T0 = témoin ; } \\
\text { diversifolia par h } \\
\text { Moyennestécar } \\
\text { probabilité après }\end{array}$ & $\begin{array}{l}\text { et les différentes } \\
\text { st du Tukey }\end{array}$ & à coté des moyennes & ctare; $T 2=6762$ & $\begin{array}{l}\text { biomasse de Tithonia } \\
\text { l'engrais composé NPK } \\
\text { ficative au seuil } 5 \% \text { de }\end{array}$ \\
\hline
\end{tabular}

Le meilleur rendement a été observé sur les parcelles à T3, c'est-à-dire celles ayant reçu 10143 $\mathrm{kg}$ de biomasse de Tithonia diversifolia par hectare comme mulch, avec un rendement moyen de 3,1 tonnes à l'hectare. Cette quantité de biomasse de Tithonia diversifolia a induit un rendement supérieur à la dose d'engrais chimique appliquée. Aucune différence significative de rendement n'a été observée entre les deux premiers niveaux de mulch de Tithonia diversifolia (T1 et T2) et la fumure minérale NPK appliquée (T4). En ce qui concerne la performance de fructification, c'est encore sur les parcelles T3 que le plus de gousses ont été produits en moyenne, bien qu'aucune différence significative n'ait été observée entre T3, T2 et T4. Cependant, par rapport à T1, il n'a pas été constaté de différence significative avec T2 et T4. Par rapport à la taille des plants, en dehors du témoin, les plantes ont affiché une croissance quasi similaire sur l'ensemble des traitements. En revanche, des nombres de gousses similaires ont été obtenus sur les parcelles ayant reçu les biomasses de Tithonia diversifolia (aux doses moyenne et faible) et celles ayant reçu les fertilisants minéraux. Bien que la faible dose de biomasse de Tithonia diversifolia puisse entrainer une faible production de gousses, elle reste néanmoins supérieure au traitement de contrôle.

Tendance du niveau de fructification et du rendement en fonction de l'application de Tithonia diversifolia: En considérant les différents traitements à base de l'apport des biomasses de Tithonia diversifolia, il ressort que la fructification du soja a connu une variation à tendance quadratique $\left(R^{2}=0,998\right)$ en fonction des quantités de biomasses appliquées (Figure 3). Une tendance similaire $\left(R^{2}=0,933\right)$ a été observée quant à l'évolution du rendement en fonction des quantités de biomasses appliquées (Figure 4). Au regard de cette tendance, il y a lieu de comprendre que l'accroissement des apports des biomasses de Tithonia diversifolia accroit la fructification et le rendement du soja jusqu'à un seuil au-delà duquel une baisse progressive peut être observée avec des apports supplémentaires de cette fumure. 


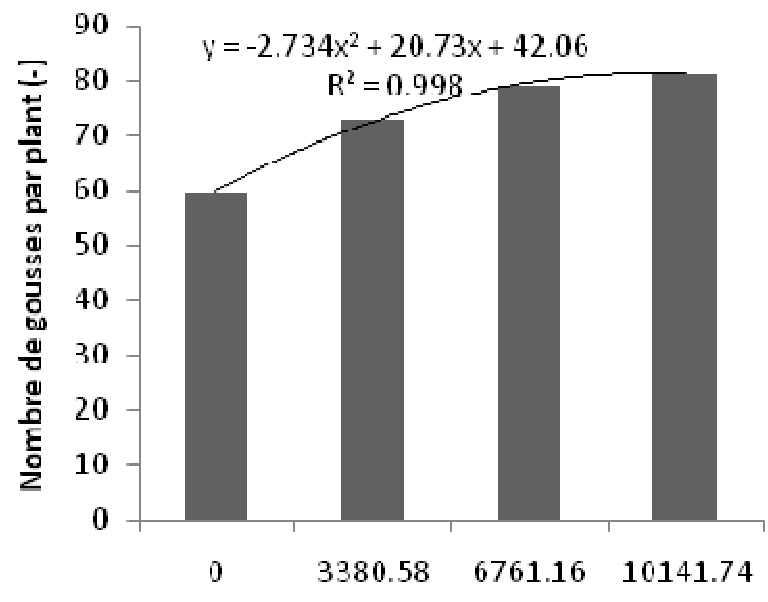

Biomasse de Tithonia diversifolio $(\mathrm{kg} / \mathrm{ha})$

Figure 3. Tendance de la fructification du soja en fonction des quantités de biomasses de Tithonia diversifolia apportées.

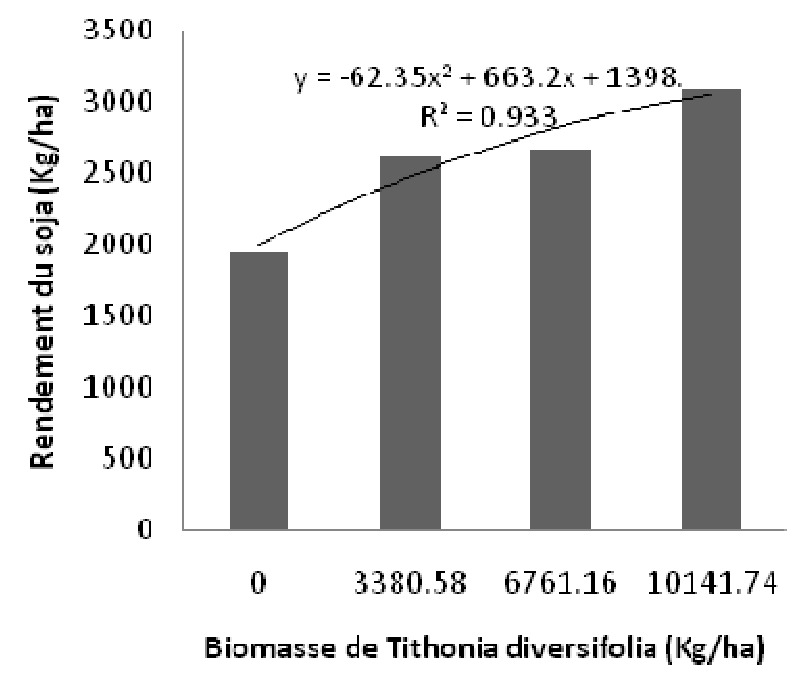

Figure 4 : Tendance du rendement du soja en fonction des quantités de biomasses de Tithonia diversifolia apportées.

\section{DISCUSSION}

Cette étude a démontré la capacité des biomasses vertes de Tithonia diversifolia à améliorer la fructification et le rendement du soja sur un sol ferralitique. Ces résultats s'apparentent à ceux obtenus dans d'autres régions d'Afrique subsaharienne, notamment par Ademiluyi et Omosoto (2007) sur le maïs au Nigeria et Kaho et al. (2011) sur la même culture au Cameroun, montrant que la tendance générale de l'évolution des propriétés de sol testées et du rendement était à la hausse par rapport au traitement témoin et à la fertilisation minérale. Les travaux de Rutunga et al. (1999) ont montré que les feuilles de Tithonia diversifolia étaient riches en $\mathrm{N}, \mathrm{K}, \mathrm{Ca}$ et $\mathrm{Mg}$. Nziguheba et al. (2002) ont trouvé que la combinaison de Tithonia diversifolia aux engrais 
inorganiques améliorait la production du maïs dans les sols pauvres en phosphore de l'ouest du Kenya. Dans le cas de cette étude, la minéralisation des biomasses de appliquées aurait induit la disponibilité des nutriments tels que le $\mathrm{N}$, le $\mathrm{K}$, le $\mathrm{Ca}$ et le $\mathrm{Mg}$, favorisant ainsi la nutrition minérale de la culture. Le traitement constitué de $10142 \mathrm{~kg}$ de biomasse de Tithonia diversifolia par hectare a même induit un accroissement de rendement supérieur à celui généré par le traitement à base de l'engrais minéral (le NPK). Appliquées en des quantités adéquates, les biomasses de Tithonia diversifolia induisent des bonnes récoltes de soja et pourraient constituer une alternative de fertilisation plus accessible aux paysans d'Afrique subsaharienne si ces derniers l'acceptaient comme fumure et en maitriser l'application. La tendance quadratique affichée par l'évolution du rendement en fonction des quantités des biomasses appliquées obéit à la loi des accroissements des rendements moins que proportionnels. Ceci revient à dire qu'on ne peut pas augmenter indéfiniment le rendement du soja en augmentant la quantité des biomasses fraiches de Tithonia diversifolia apportées au sol. Par contre, la quantité optimale des biomasses à apporter au sol peut être déterminée par la dérivée première de l'équation de la courbe de tendance. L'utilisation des engrais commerciaux en agriculture paysanne a généralement été restreinte seulement à quelques exploitations dotées des ressources telles que les vaches, la terre (Shepherd et Soule, 1998), et avec des revenus extra agricoles élevés (Niang et al., 1998). La faible production des sols témoins peut être attribuée aux facteurs caractéristiques des sols acides : $\mathrm{pH}$ acide, toxicité $\mathrm{Al}$ et $\mathrm{Mg}$, déficiences en nutriments (Ca, Mg, P, K, B et Zn) (Mulaji, 2010). En plus, sur les parcelles témoins, l'absence d'apports organiques s'accompagne d'une perte en matières organiques et en nutriments, d'une acidification des sols, d'une réduction de la biomasse et de l'activité microbienne, d'une insolubilisation du phosphore qui ensemble contribuent à la baisse sensible des rendements des cultures (Deblay, 2006). Les variabilités constatées entre les doses de biomasses vertes de Tithonia diversifolia pourraient s'expliquer par les interactions entre les éléments minéraux et le $\mathrm{C} / \mathrm{N}$. Par ailleurs, le traitement à la plus forte dose de biomasse verte de Tithonia diversifolia a donné des résultats supérieurs à ceux obtenus avec les fertilisants inorganiques d'une part et la dose moyenne et faible de biomasse verte de Tithonia diversifolia. Cela semble suggérer que la synchronisation de la libération des éléments nutritifs par la biomasse de Tithonia diversifolia pendant leur décomposition et leur assimilation par la plante test était bonne. En effet, Palm (1995) et Cobo et al. (2002) ont montré que le taux de décomposition de la matière organique et l'augmentation des rendements étaient étroitement lié à la synchronisation entre la libération des nutriments et leur assimilation par la plante. La biomasse verte de Tithonia diversifolia incorporée dans le ferralsol semble donc avoir un taux de décomposition convenable qui a permis à la plante d'assimiler une grande proportion des nutriments libérés lors de la décomposition de la matière organique. Des résultats similaires obtenus au Kenya par Muna- Mucheru et al. (2007) ont montré que les parcelles ayant reçu la biomasse de Tithonia diversifolia (avec ou sans engrais chimiques) ont donné des rendements de maïs les plus élevés (5,5 et 5,4 tha ${ }^{-1}$ respectivement). De même, Jama et al. (2000) et Kaho et al. (2011) ont rapporté que les rendements en grain de maïs étaient supérieurs sur les parcelles de Tithonia diversifolia seul comparés aux parcelles ayant reçu seulement les engrais chimiques. En plus, dans les conditions de la région de Lubumbashi, Useni et al. (2012) ont obtenu des résultats similaires sur la culture de mais avec utilisation de biodéchets à base de fumiers de poules. II est aussi établi dans la littérature que les sols déficients en azote sont susceptibles de répondre mieux à l'application des engrais organiques (Kaho et al, 2011). Par ailleurs, la supériorité de la biomasse verte de Tithonia diversifolia par rapport aux engrais minéraux, malgré leur libération rapide des éléments minéraux, serait liée à l'amélioration des propriétés des sols couplée à la fourniture des 
éléments minéraux. En effet, les engrais minéraux jouent le rôle trophique alors que les amendements organiques jouent le rôle trophique

\section{CONCLUSION}

Les résultats de cette étude ont montré que les biomasses fraiches de Tithonia diversifolia, améliorent la fertilité du sol et augmentent de façon significative les rendements du soja. Dans les conditions de cet essai, Tithonia présente un grand potentiel pour l'amélioration de la disponibilité des éléments nutritifs sur un ferralsol sol et peut fournir les quantités des nutriments nécessaires à la culture de soja. En outre, les

\section{RÉFÉRENCES BIBLIOGRAPHIQUES}

Ademiluyi, B.O. \& Omotoso, S.O., 2007. Comparative Evaluation of Tithonia diversifolia and NPK Fertilizer for soil improvement in maize (Zea mays) production in Ado Ekiti, Southwestern Nigeria, Am.-Eurasian J. Sustain. Agric., 1(1): 32-36

Cobo J.G., Barrios E., Kaas D.C.L \& Thomas R.J., 2002. Nitrogen mineralization and crop uptake from surface-applied leaves of green manure species on a tropical volcanic-ash soil. Biology and fertility of soils, 36: 87- 92.

Chukwuka K.S. \& Onatayo O.E., 2009. Soil fertility restoration potentials of Tithonia green manure and water hyacinth compost on a nutrient depleted soil in south western Nigeria using Zea mays L. as test crop. Research journal of soil biology 1 (1): 2030

Deblay S., 2006. Fertilisation et amendements. Educagri éditions, 2ème édition, Paris, 129 p.

FAO, 2005. New_LocClim: Local Climate Estimator. FAO Environment and Natural Resources Working Paper, $\mathrm{N}^{\circ} 20$.

Ganunga R., Yerokun O. \& Kumwenda J.D.T., 1998. Tithonia diversifolia: an organic source of nitrogen and phosphorus for maize in Malawi. In: Waddington SR et al. et améliorent en même temps les propriétés du sol (Mulaji, 2010).

rendements obtenus avec les doses croissantes de biomasse vertes de Tithonia diversifolia, sont de loin supérieurs à ceux obtenus sur le témoin et les parcelles avec engrais minéraux NPK 10-2010 , ce qui est capital pour les petits agriculteurs de la zone d'étude. D'autres études sont cependant, nécessaires pour évaluer la rentabilité de différentes doses de biomasses vertes de Tithonia diversifolia.

(eds) Soil Fertility Research for MaizeBased Farming Systems in Malawi and Zimbabwe, pp 191-194. Soil Fert Net and CIMMYT-Zimbabwe, Harare, Zimbabwe

Jama B., Palm C.A., Buresh R.J., Niang A.I., Gachengo C. \& Nziguheba G., 2000. Tithonia as a green manure for soil fertility improvement in Western Kenya: a review. Agroforestry Systems, 49: 201-221.

Javaheri F., Baudoin J.P., 2001. Le soja. in Raemaerkers R.H (ed).Agriculture en Afrique Tropical (Directorate General for International Cooperation), Ministry of Foreign Affairs, External Trade and International Cooperation, Brussels, Belgium.p860-882.

Jiri O. \& Waddington S.R., 1998. Leaf prunings from two species of Tithonia raise maize grain yield in Zimbabwe, but take a lot of labor! Newsletter of Soil Fert Net, Harare, Zimbabwe. Target 16: 4-5

Kaho F., Yemefack M., Feujio-Tegwefouet P. \& Tchanthaouang J.C., 2011. Effet combiné de feuilles de Tithonia diversifolia et des engrais inorganiques sur le rendement du maïs et les propriétés d'un sol ferralitique au centre du Cameroun. Tropicultura, 29 (1): $39-45$

Mbonigaba M.J.J., 2007. Étude de l'impact des composts à base de biomasse végétale 
sur la dynamique des indicateurs physicochimiques, chimiques et microbiologiques de la fertilité des sols: application sur trios sols acides tropicaux du Rwanda. Thèse de doctorat, FUSAGx, Gembloux, 243p.

Mulaji K.C., 2010. Utilisation des composts de biodéchets ménagers pour l'amélioration de la fertilité des sols acides de la province de Kinshasa (République Démocratique $d u$ Congo). Thèse de doctorat, universite de Liège- Gembloux Agro-Biotech, 220p.

Muna-Mucheru M., Mugendi D., Kung'u J., Mugwe J. \& Bationo A., 2007. Effects of organic manure and mineral fertilizer inputs on maize yield and soil chemical properties in a maize cropping system in Meru South District, Kenya, Agroforestry Systems, 69:189-197.

Niang A., Amadalo B., Gathumbi S. \& Obonyo C.O., 1996. Maize yield response to green manure application from selected shrubs and tree species in western Kenya: a preliminary assessment. In: Mugah JO (ed) Proceedings of the First Kenya Agroforestry Conference on People and Institutional Participation in Agroforestry for Sustainable Development, pp 350-358. Kenya Forestry Research Institute (KEFRI), Muguga, Kenya

Niang A., De Wolf J., Hansen T., Nyasimi M., Rommelse R. \& Mwendwa K., 1998. Soil fertility replenishment and recapitalization project in western Kenya. Progress report, February 1997-July 1998. Pilot Project Report No. 8. Regional Agroforestry Research Centre, Maseno, Kenya

Nziguheba G., Palm C.A., Buresh R.J. \& Smithson P.C., 1998. Soil phosphorus fractions and absorption as affected by organic and inorganic sources. Plant and Soil, 198: 159-168

Rutunga V., Steiner K.G., Karanja K.N., Gachene C.K.K. \& Nzabonihankuye G., 1998. Continuous fertilization on non-humiferous acid Oxisols in Rwanda "Plateau Central": Soil chemical changes and plant production. Biotechnol. Agron. Soc. Environ. 2 (2):135-142.

Shepherd D.K. \& Soule, J.M., 1998. Soil fertility management in western Kenya: dynamic simulation of productivity and sustainability at different resource endowment levels. Agric. Ecosyst. Environ. 71:131-145.

Useni S.Y., Baboy L.L., Nyembo K.L. \& Mpundu M.M., 2012. Effets des apports combinés de biodéchets et de fertilisants inorganiques sur le rendement de trois variétés de Zea mays L. cultivées dans la région de Lubumbashi. Journal of Applied Biosciences 54: 3935- 3943 